ERRATUM TO

\title{
ASYMPTOTIC AND CONVERGENT FACTORIAL SERIES IN THE SOLUTION OF LINEAR ORDINARY DIFFERENTIAL EQUATIONS
}

\author{
ROBERT L. EVANS
}

(Published in the Proceedings of the American Mathematical Society vol. 5 (1954) pp. 89-92.)

An error in the proof of the entitled article was kindly pointed out by Professor H. L. Turrittin; its correction introduces a limitation on the argument of $\omega$ in Theorem 1. To outline the correction in terms consistent with those of the article, let $v_{\nu}(z)$ correspond to a segment on the Puiseux diagram for (1) extending from $\left(\beta_{1}, m\left(\beta_{1}\right)\right)$ to $\left(\beta_{1}+N\right.$, $\left.m\left(\beta_{1}+N\right)\right)$ - so that $s_{0}=\left(m\left(\beta_{1}+N\right)-m\left(\beta_{1}\right)\right) / N$ - and call

$$
p_{\beta_{1},-m\left(\beta_{1}\right)} h^{N}+\cdots+p_{\beta_{1}+N,-m\left(\beta_{1}+N\right)}(h)^{0}=0
$$

the characteristic equation. Thus (11) has one root $h_{v}$ which relates to the considered particular solution. Substitutions (4) and (5) convert (1) to a differential equation for $v_{\nu}(z)$ in which the analogue of (11) has roots $\left(h_{1}-h_{v}\right), \cdots,\left(h_{N}-h_{v}\right)$ if $h_{1}, \cdots, h_{N}$ are the roots of (11). For definiteness, let $m$ of (4) be the least positive integer making $\left(m s_{0}\right)$ an integer or 0 . Then the $a_{\nu, \mu}$ 's of (3) and later equations are defined as the product of a gamma function and the solution of a difference equation of Poincaré type (reference of footnote 6, chap. XVII), and the characteristic equation for this difference equation has $\left(h_{1}-h_{\nu}\right)^{-1 / m\left(s_{0}+1\right)}, \cdots,\left(h_{N}-h_{\nu}\right)^{-1 / m\left(s_{0}+1\right)}$ as its roots. The $a_{\nu, \mu}$ 's are a particular solution for which $\lim _{\mu \rightarrow \infty}\left(a_{\nu, \mu+m\left(s_{0}+1\right)} / a_{\nu, \mu}\right)$ is one of $\left(h_{1}-h_{\nu}\right)^{-1}, \cdots$, or $\left(h_{N}-h_{\nu}\right)^{-1}$, if it exists-we conjecture that this limit is one value among those of $\left(h_{1}-h_{\nu}\right)^{-1}, \cdots$, and $\left(h_{N}-h_{\nu}\right)^{-1}$ having the largest modulus. If $\alpha$ represents the argument of this limit we have, as a final result, that the convergence in Theorem 1 necessitates the additional restriction on arg $\omega$ :

$$
\begin{aligned}
\left|\arg \omega-(\alpha+M \pi) / m\left(s_{0}+1\right)\right|< & \pi / 2 m\left|s_{0}+1\right| \\
& \text { (where } M=\text { odd integer). }
\end{aligned}
$$

